\title{
Empirical Study on Reversible Lane in Beijing
}

\author{
X. Wang, Y.L. Wang, M.C. Zhang \\ Beijing City University \\ China
}

\begin{abstract}
Setting the reversible lane can make full use of the existing road resources and effectively relieve urban roads tidal traffic problems during peak hours. Analysis of characteristics of tidal traffic phenomena and causes. Elaborating the related concepts of reversible lane traffic organization and application from road conditions and traffic flow characteristic. By evaluation of the Chaoyang Road reversible lane and analysis the feasibility of Litang Road reversible lane. Finally through vissim simulation results show that reversible lane is practical and feasible, significance for popularization.
\end{abstract}

Keywords-tidal traffic; traffic jam; reversible lane; traffic organization;vissim.

\section{INTRODUCTION}

At present, large and medium-sized city roads, especially in the morning and evening rush hours, traffic flow for work or school shows normalization of the "tidal" distribution, which results in the same road or crossing, at different time in the day, the phenomenon of uneven use of road resources. In this paper, the research results show that reversible lane strategy is an effective approach to deal with the road usage imbalance due to tidal traffic.

\section{CHARACTERISTICS OF THE TIDAL TRAFFIC IN THE MORNING AND EVENING RUSH HOURS}

Due to the heavy traffic road resources grossly inadequate in rush hours and other times with the traffic smooth, part of the road appears the phenomenon of wasting resources, so in most cases this change cannot implement fixed one-way traffic in the whole day.

\section{A. Reasons Of The Tidal Traffic}

Throughout the large and medium-sized cities, traffic problems is triggered by the urban social aspects of cause, which is influenced by city planning, price regulation, the transportation development policy, etc. Up to $20-30 \%$ of roads and intersections exist tidal flow distribution, tidal traffic produces the main reason:

1. With the expansion of city scales, urban land value rising, the house prices stubbornly high, a large number of urban residents have to live in suburbs; however, their workplaces still remain in central city. As a result, the commuting traffic presents tidal feature.

2. Frequent use of household cars contributes to tidal traffic based on the commuting traffic. With the improvement of residents' income level in our country, the car as a motorized traffic tool, becomes one of the main ways of resident trip. But the use of a large number of household cars, bringing the convenience to the residents, this also causes more traffic problems, especially creating greater pressure to tidal traffic.

3. Traffic flow because of holidays, large-scale activities is also one of the reasons for the tidal traffic, especially in big cities. Tourism shopping, visiting friends and other activities lead traffic flow to a sharp rise in a short time. Combined with the implementation of small car toll free scheme in holidays, the expressway segment near the city is easy to form the tidal traffic at the beginning and end of the holidays.

\section{B. Characteristics of The Tidal Traffic}

Through the analysis of the forming factors of tidal traffic, it has the following features:

1. The most remarkable characteristic of tidal traffic is the direction and flow of tidal traffic would obvious change along with the time changing.

2. The common characteristic of tidal traffic is road space resources utilization rate is low usually.

3. The tidal traffic has obvious periodicity and regularity.

4. Sudden tidal traffic of holidays, large gatherings has some sudden and sharp.

\section{TRAFFIC ORGANIZATION OF REVERSIBLE LANE}

\section{A. Reversible Lane}

Reversible lane refers to a kind of traffic organization that some lanes can change the direction or traffic type in the different time. It mainly aims at the rush hours of tidal traffic, in the existing road infrastructure supply unchanged, by changing the direction of the lane or traffic type to adapt to the actual traffic demand, so as to improve the efficiency of road usage, to reduce the queue length and to lower the average unit vehicle delay[1].

According to the different role, this kind of lane can be divided into two categories:

1. Reversible lane: the direction would be changed in different time, also called variable lane.

2. Other reversible lane: some kinds of lane traffic would be adjusted in different time, such as non- motor vehicle lane into motor lane, to improve the efficiency of the overall travel.

This paper discussed is application of reversible lane.

\section{B. Setting Conditions Of Reversible Lane}

According to the reversible lane setting conditions recommended by the American Institute of Transportation Engineers(ITE) need to meet the following several aspects: 
1. The number of motor vehicle lanes should be more than 3 for the two-way road, at least 5; and there is no central isolation belt or tram rail.

2. The $\mathrm{Kd}$ reflects the imbalance of traffic flow in two directions. The Kd should be larger than 2/3;

3. After setting reversible lane, each direction capacity should be meet the new traffic demand respectively, especially reducing Lane direction;

4. After setting reversible lane, the number of Lane guidance should be increased in signal control intersection.

5. Determining the starting and ending point of reversible lane should consider the change of traffic flow, and as far as possible to consider the ability of traffic diversion ability at the origin and the destination.

\section{Timing Of Switch Reversible Lane}

Rationally determining the reversible lane switching time is the premise of giving full play to the function of reversible lane, improving traffic capacity, so must be full of prophase investigation.

1. Generally, in the morning and evening rush hours, the main roads into and out of city have high flow and clear direction imbalance. By collating the road traffic data, drawing the regularities of tidal traffic, determining the beginning and ending time of rush hours, general early in this moment to switch the reversible lane. Taking Beijing area as an example, the morning peak generally appears in the 7:00-9:00 and evening peak in 17:00-19:00. Removing rush hours, the reversible lane must be restored the original direction, and not be disposed in a single direction of travel.

2. Because the distance between adjacent intersections is smaller, conflict point of mixed traffic is more, Non-motor vehicles and pedestrians often cross the road at the same time, as the start condition of reversible lane heavy traffic direction saturation can be reached more than 0.8.

3 . When the $\mathrm{Kd}$ is close to $2 / 3$, the difference between the two directions is quite serious. Therefore, the $\mathrm{Kd}$ reaches $2 / 3$ opening the reversible lane and below 2/3 closing it.

It should be noted that, when setting the tide lane, it will increase the amount of construction of corresponding facilities and $\mathrm{f}$ traffic management. And the driver must focus on traffic information provided by traffic signs.

\section{Setting Scheme Of Reversible Lane}

Up to today the absence of standard reversible lane scheme in China, learning from the existing setting principles and experience, such as Manual on Uniform Traffic Control Devices[4] (MUTCD), the setting scheme includes the following main steps[2]:

1. Reconstruction of road infrastructure. Demolition of existing fixed central isolation facilities ,delimit signs marking, additional lane sign to control the switching time ,setting reversible lane signs at a distance to prompt reversible lane information repeatedly, at the same time with supplemented text.
2. Formulating operation scheme. According to the actual situation of road, formulating the number of reversible lane, lane direction, Switching time. If there are many intersections in the reversible lane, for security reasons, it is the best to fit with no left turn, no U-turn and other measures, while giving the bypass scheme.

3. Evaluating the scheme. After a period of implementation, it should to evaluate and optimization the scheme, such as adjusting the switch time, reversible lane length.

\section{E. Application Of Reversible Lane}

The technology of reversible lane has almost 80 years of research and practice, what is one of the commonly used control ways in foreign cities. In America, Golden Gate Bridge in San Francisco, is two-way road with 6 lanes. One lane has be adjusted using the separation guardrail in the middle to each side according to a preset time point. As early as 1928 reversible lane system was applied on 8th road in Los Angeles, traffic cones and signs were applied to control reversible lane on Will Hill avenue in1937. In England, Bermingham, Calgary -- Center Street, Maryland have reversible lanes[3].

In China there are many cities using reversible lane[4]. The first reversible lane in 2004 was opened in Shenyang, and after the success Shenyang gradually extended this model within the city. The outer ring tunnel in Shanghai has reversible lane by variable isolation guardrail. Shenzhen Meilin Guan, Chongqing city Yuzhong District CBD important roads Cotton Street have reversible lanes. Beijing has two reversible lanes, Chaoyang road and Zizhuyuan Road. The former was opened in September, 2013, the latter in September, 2014.Since the latter has just been opened, the former has been regarded as the key to the study and analysis in this paper.

\section{EVALUATION OF THE CHAOYANG ROAD REVERSIBLE LANE IN BEIJING}

Chaoyang Road is a main radial line in the eastern part of Beijing, which connects central urban and east localities, especial Tongzhou District. So the tidal traffic is more Prominent.

\section{A. Description Of Chaoyang Road Reversible Lane}

This reversible lane originates from the intersection of Chaoyang Road - Jing Guang Bridge, East 3rd Ring Road, and in the west terminates in the 150 metres west of Ciyunsi Bridge, East 4rd Ring Road, with a length of $2.3 \mathrm{~km}$.

1.Characteristics of Chaoyang Road tidal traffic and road conditions

It is a two-way road selected to setting reversible lane with 8lanes, the main road of which is the two-way with 4 lanes, including two BRT lanes, two bus lanes and the side road of which is two-way with 6 Lane. There is no isolation belt between the main and side road, meeting the conditions of reversible lane. As one of the city main roads, the design speed for Chaoyang Road is $60 \mathrm{~km} / \mathrm{h}$ and the theory road capacity is $1730 \mathrm{pcu} / \mathrm{h}$ in a lane. Taking the impact of lane width, multilanes, intersections and mixed traffic and so on into consideration, the possible road capacity can meet the needs of traffic. This road selected to setting reversible lane was more 
T-intersections, connecting the roadside residential area , which is no impact on the reversible lane. It is only one large in 9 intersections, the intersection of Xidawang Road Chaoyang Road. The traffic flow survey time was 8:00-9:00 and 18:00-19:00, June 10, 2013 , as the investigation sites link between 3rd Ring Road and 4rd Ring Road, shown in table 1.

TABLE I. STATISTICS OF TRAFFIC FLOW OF CHAOYANG ROAD IN JUNE 10, 2013.

\begin{tabular}{|l|l|l|l|l|}
\hline & $\begin{array}{l}\text { 8:00-9: 00 } \\
\text { (the morning rush } \\
\text { hours) }\end{array}$ & \multicolumn{2}{|l|}{$\begin{array}{l}\text { 18:00-19:00 } \\
\text { (the evening rush hours) }\end{array}$} \\
\cline { 2 - 5 } & $\begin{array}{l}\text { West-East } \\
\text { (out of } \\
\text { city) }\end{array}$ & $\begin{array}{l}\text { East- } \\
\text { West } \\
\text { (into of } \\
\text { city) }\end{array}$ & $\begin{array}{l}\text { West-East } \\
\text { (out of city) }\end{array}$ & $\begin{array}{l}\text { East- West } \\
\text { (into of } \\
\text { city) }\end{array}$ \\
\hline $\begin{array}{l}\text { traffic } \\
\text { flow(pcu/h) }\end{array}$ & 1520 & 1858 & 2640 & 1360 \\
\hline Kd & 0.45 & 0.55 & 0.66 & 0.34 \\
\hline
\end{tabular}

According to the investigation data, although two direction flow of Chaoyang Road are difference in the morning rush hours, there are not very different. While in the evening rush hours traffic flow out of city was greater than into of city, basically conforming to the reversible lane conditions. In addition, the small peak into of city may also appear at noon, if the lane does not change back to the normal direction, it will cause traffic congestion.

\section{Setting scheme of Chaoyang Road reversible lane}

The specific scheme is that the Innermost lane into of city would be changed for out of city, from $17 \mathrm{pm}$ to $20 \mathrm{pm}$ and Other times unchanged. This means that main road out of city increases a lane in evening rush hour. So that the four-lane plays six lanes function. The transportation administration has set up 16 gantries in main road from the origin to the destination of the reversible lane, a sign above each lane. The white arrow lighting indicates that the lane can pass, while the red cross lighting indicates that the lane prohibited. In addition, due to cannot delimit guide markings and stop line in the approach road of the lane, variable isolated guardrails have been installed to ensure safety. In everyday 5-10 minutes before switching reversible lane, the message board in the front $350-400 \mathrm{~m}$ of it starts to prompt vehicles to the outward lane. When the sign (white arrow/red cross) lighting, the police car will drive from the origin to the destination and move Variable guardrail to the left or right, in order to change the lane direction. The whole process can be carried out video surveillance.

When cars turning left from south to west, or turning right from north to west, or U-turn in the west, must first drive into the north side road. At the same time with traffic flow into of city increasing, it should to make the divergence measure. Such as vehicles into of city can bypass Chaoyang North Road, Chaoyang park South Road in the north, or Jianguo Road, Tonghui River Road in the south, to alleviate the pressure due to reducing one lane.

With the increase of a reversible lane, it is expected that traffic speed into of city will rise somewhat, the traffic speed must be under $70 \mathrm{~km} / \mathrm{h}$ in reversible lane .

\section{B. The Simulation Evaluation Of Chaoyang Road Reversible Lane}

As the Beijing first reversible lane, it has achieved good effect after implementing more than a year. Based on the survey data in this paper, combined with the characteristics of traffic flow, using Vissim software simulate and evaluate traffic flow status before and after the reversible lane setting, the specific results are shown in Fig. 1. It is thus obvious to improve the possible road capacity of the out the city by $25.3 \%$, increase the average travel speed by $7.50 \%$, reduce the average unit vehicle delay by $15.25 \%$, and improve the traffic condition in road network conspicuously.

\section{ANALYSIS THE FEASIBILITY OF LITANG ROAD REVERSIBLE LANE IN BEIJING}

Litang Road is a main road of the Beijing northern region. It is the main channel in and out of the city such as Tiantongyuan, Huilongguan which are the large residential areas. The road also has the typical tidal traffic phenomena.

According to the field survey, Litang Road has a two-way road with 8 lanes, the main road of which is the two-way with 6 lanes. The main road has 0.8 meters central isolation belt, and main and auxiliary road is separated by green belt. The traffic flow survey time was 8:00-9:00 and 18:00-19:00, June 17, 2013, the investigation sites were Lishui Bridge subway near Tiantongyuan, shown in table

TABLE II. STATISTICS OF TRAFFIC FLOW OF LITANG ROAD IN JUNE 17, 2013.

\begin{tabular}{|l|l|l|c|c|}
\hline & $\begin{array}{l}\text { 8:00-9: 00 } \\
\text { (the morning rush } \\
\text { hours) }\end{array}$ & $\begin{array}{l}\text { 18:00-19:00 } \\
\text { (the evening rush hours) }\end{array}$ \\
\cline { 2 - 5 } & $\begin{array}{l}\text { South- } \\
\text { North } \\
\text { (out of } \\
\text { city) }\end{array}$ & $\begin{array}{l}\text { North- } \\
\text { South } \\
\text { (into of } \\
\text { city) }\end{array}$ & $\begin{array}{l}\text { South-North } \\
\text { (out of city) }\end{array}$ & $\begin{array}{l}\text { North- } \\
\text { South } \\
\text { (into of } \\
\text { city) }\end{array}$ \\
\hline $\begin{array}{l}\text { traffic } \\
\text { flow(pcu/h) }\end{array}$ & 3145 & 4940 & 3150 & 2072 \\
\hline Kd & 0.39 & 0.61 & 0.60 & 0.40 \\
\hline
\end{tabular}

So the traffic flow into of city was significantly greater than out of city in the morning rush hours, especially for household car, the traffic flow into of city of total traffic flow is $61 \%$. On the contrary, the traffic flow out the city of total traffic flow is $59 \%$ in the evening rush hours. It Complies with the basic conditions for reversible lane. Obtained by the simulation and comparison, it is thus obvious to increase the possible road capacity of the out of city by $15.3 \%$, reduce the average unit vehicle delay by $46 \%$, shown in Fig.2. according to the scheme of Litang Road reversible lane, cancel the central isolation belt of the main road, set the Litang Road reversible lane. 


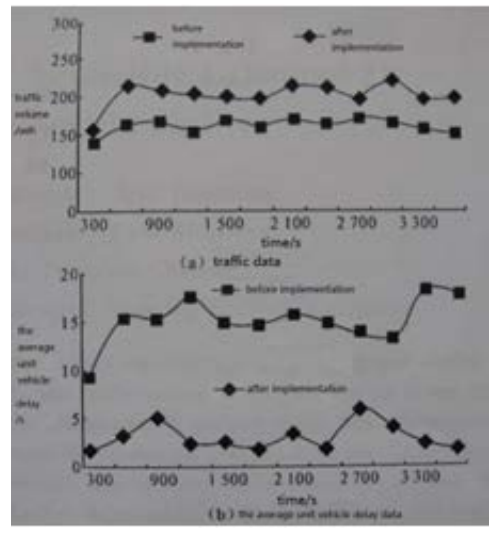

FIGURE I. EFFECT COMPARISON BEFORE AND AFTER IMPLEMENTATION OF CHAOYANG ROAD REVERSIBLE LANE.

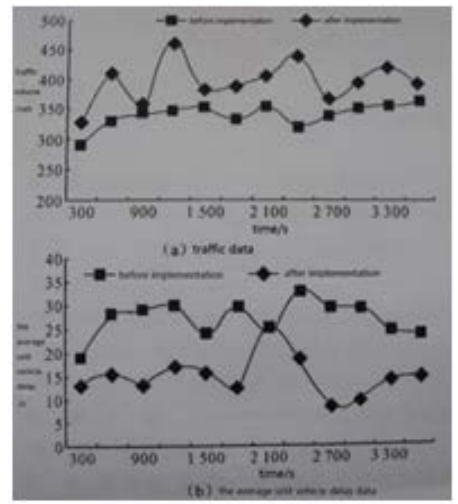

FIGURE II. EFFECT COMPARISON BEFORE AND AFTER ASSUMED LITANG ROAD REVERSIBLE LANE.

\section{CONCLUSIONS}

Although improving the average unit vehicle speed, the traffic light timing is not reasonable, frequent changing lane within a short distance, vehicles shunting to the auxiliary road increasing, traffic congestion has not been fundamentally resolved. It should to strengthen investigate and survey, timely extening the reversible lane and reasonable setting traffic lights.

\section{REFERENCES}

[1] G.H. Zhang, Study on the Setting of Reversible Lane in Urban Road Engineering, No.3 in Transportation Science and Technology, pp128133, 2012.

[2] L.L. Dai, J.G. Gu, C.J. Yu and H.T. Qiu, Traffic Flow Characteristics on Reversible Lane and Its Operational Plan Based on Simulation, No.1 in Journal of Transport Information and Safety,pp59—63, 2012.

[3] X. Liang, C.P. Hu, C.Y. Ma and B.H. Mao, Empirical Study on Variable Lanes Design of Chaoyang North Street in Beijing, 22-24 in Proceedings of the 30th Chinese Control Conference, pp5527-5531, 2012.

[4] H.L. Xu, Q. Yu, Present Situation and Prospect of Variable Lane Research at Home and Abroad, No.15,Vol.42 in Transportation Standardization, pp64—67, 2014. 